\title{
Growth dynamics of pulsed laser deposited nanofoams
}

\author{
A. Maffini,,${ }^{1,}$ A. Pazzaglia, ${ }^{1}$ D. Dellasega, ${ }^{1,2}$ V. Russo, ${ }^{1}$ and M. Passoni ${ }^{1,2}$ \\ ${ }^{1}$ Dipartimento di Energia, Politecnico di Milano, Italy \\ ${ }^{2}$ Istituto di Fisica del Plasma “P. Caldirola”, CNR, via R. Cozzi 53, 20125 Milano, Italy
}

(Received 22 January 2019; published 30 August 2019)

\begin{abstract}
We present a modeling and experimental investigation about the physics of nanofoam growth in pulsed laser deposition (PLD) experiments. Thanks to their unique features, ultralow density materials-known as nanofoams-are attracting a growing interest for many cutting-edge applications. PLD has emerged as one of the most promising and versatile techniques for the synthesis of nanofoam; however, the lack of a satisfactory comprehension of the nanofoam growth process hinders the unleashing of their full potential as innovative materials. In this work we propose a snowfall-like model that describes the nanofoam growth as the coalescence of micrometric-sized fractal aggregates of nanoparticles, where the aggregates are formed through an in-fight process whose timescale is determined by the shot-to-shot time interval. We exploit these insights to demonstrate an approach to control the nanofoam properties down to the nanoscale. These results, along with their interest from a fundamental point of view, open perspectives in the field of low density nanostructured materials.
\end{abstract}

DOI: 10.1103/PhysRevMaterials.3.083404

\section{INTRODUCTION}

Ultralow density nanostructured materials, also known as "nanofoam," constitute a broad class of materials characterized by a large fraction of submicrometric voids and/or pores (typically $\geqslant 90 \%$ ). Nanofoams exhibit ultralow density [1] $\left(1-100 \mathrm{mg} / \mathrm{cm}^{3}\right)$ and extremely high surface-to-volume ratio, which make them of interest for many research fields of great societal and technological importance, such as hydrogen storage [2], next generation supercapacitors [3] and catalysts [4,5], water treatment [6], gas sensing [7], and medicine [8]. In the past years, nanofoam materials derived from different elements have been investigated, namely noble metals [2,9], transition metals [3-5], oxides [10,11], semiconductors [12], and organic compounds. Among them, carbon (C) nanofoams have attracted an impressive amount of research efforts because of their unusual and appealing features, like an unconventional ferromagnetic behavior [13,14], giant optical absorption [15], and an increased adsorption and storage capability [16], together with the unique carbon capability of forming chemical bonds that are very different in their nature (i.e., $s p^{1}, s p^{2}$, and $s p^{3}$ hybridization). Several techniques have been proposed for the synthesis of $\mathrm{C}$ nanofoam (see for example [17] and references therein). Among them, pulsed laser deposition (PLD) has emerged as an ideal tool to produce ultralow density $\mathrm{C}$ nanofoam since the seminal work done by Rode and co-workers [18]. PLD is a widely used material production technique, is versatile in a broad range of tunable process parameters, and allows to use virtually every kind of substrates. It has been demonstrated that PLD can be exploited to produce $\mathrm{C}$ nanofoams with controlled and reproducible mean density and thickness [13,18-23].

\footnotetext{
*alessandro.maffini@polimi.it
}

These aspects can be of crucial importance also in the light of specific applications. An example is given by the development of advanced target concepts for superintense laser-driven ion acceleration, where an ultralow density layer ( $\simeq 10$ 's of $\mathrm{mg} / \mathrm{cm}^{3}$ ) is attached to a micrometric solid foil (see for example [24] and references therein). In previous works we have shown that PLD can be exploited to grow carbon nanofoam suitable as a low-density layer in advanced targets for laser-driven ion acceleration [25-28]. We have also observed that the nanofoam morphology at the micro- and nanoscale plays a major role in determining the effectiveness of the laser acceleration process [29] and hence a precise morphological control of the pulsed laser deposited nanofoam is highly desirable.

It is well understood that PLD carbon nanofoams are essentially void-rich, fractal-like structures made of carbon nanoparticles [30], with an average diameter ranging from a few nanometers up to tens of nanometers [7,18,19,21,31]. Production of nanoparticles by pulsed laser ablation has been observed both in nanosecond [32-34] and subpicosecond regimes [35-37]. Generally speaking, nanoparticles are formed in the first stages of laser ablation process either by a direct ejection from the target (typically in the case of subpicosecond laser pulses) or during the expansion of the ablated species in a background atmosphere. Different theoretical models describing the synthesis of nanoparticles have been proposed [38-41]; remarkably, it has been shown that the generation of nanoparticles represents a general feature of laser ablation and it is not limited to specific materials or deposition regimes [42,43].

In contrast with the rich and vast body of literature describing the synthesis of nanoparticles in laser ablation experiments, the physics of how the nanoparticles spatially arrange themselves to give rise to the peculiar nanofoam morphology, i.e., the physics of nanoparticle aggregation which determines 
the growth of nanofoam films, is poorly understood. Only a few articles are devoted to the study of fractal structures resulting from the coalescence of laser-ablated nanoparticles with various elemental compositions [44-47]. In these works, two main aggregation mechanisms are proposed to explain the morphological features of the deposited structures, namely nanoparticle diffusion on substrate $[46,47]$ and nanoparticle aggregation occurring in flight [44]. However, a complete and satisfactory picture of the various processes (and the corresponding timescales) that take place during the PLD process is still lacking, and many questions regarding the relation between material properties (e.g., average density and average aggregate size) and PLD parameters are not answered yet. This aspect is even more relevant considering that nanofoam obtained by nanosecond PLD are grown exploiting an unconventional, largely unexplored PLD regime characterized by very high background pressure (up to $1000 \mathrm{~Pa}$ ), in which nanofoam properties could be influenced also by process parameters that are not usually investigated in conventional, low-pressure PLD regimes. A deeper and better comprehension of nanofoam growth dynamics-besides its interest from a fundamental and cross-disciplinary point of view-is therefore crucial to unleash the full potential of PLD as a versatile tool for the production of advanced ultralow density materials.

In this paper we propose an experimental and modeling investigation of the physics underlying the growth of $\mathrm{C}$ nanofoam in nanosecond PLD experiments. In particular, we focus on the dynamics of the nanoparticle aggregation into a foamlike structure, while the synthesis of nanoparticles is not discussed here. After having presented the methods and tools that we use in this work, we study the evolution of nanofoam morphology through different stages of growth, characterized by an increasing number of laser shots. Having shown that the peculiar nanofoam structure is given by the coalescence of aggregates in a snowfall-like process, we correlate the aggregate size with the aggregation timescale. We develop two alternative models to describe the relationship between the aggregation timescale and the PLD parameters, and we test them experimentally. Finally, we exploit the insights gained about the aggregation timescale to demonstrate how it is possible to control nanofoam morphology and density by acting on the laser repetition rate.

\section{METHODS}

In a typical nanosecond PLD experiment, a pulsed laser beam is focused on a solid target with a repetition rate which is the inverse of the shot-to-shot time $T_{\text {sts }}$, causing the evaporation of its superficial layers. The ablated species expand in a vacuum chamber that can be filled with a background gas at a desired pressure, and finally impinge on a substrate placed at a variable distance $d_{\mathrm{ts}}$ from the target. The ablated species are slowed down and confined by the interaction with the background gas, potentially leading to the formation of atomic clusters and nanoparticles. By acting on the background gas pressure one can control the nature of the species arriving on the substrate (either atoms, atomic clusters, or nanoparticles), their kinetic energy, and, in the case of nanoparticles, their aggregation dynamics. These features, in turn, determine the

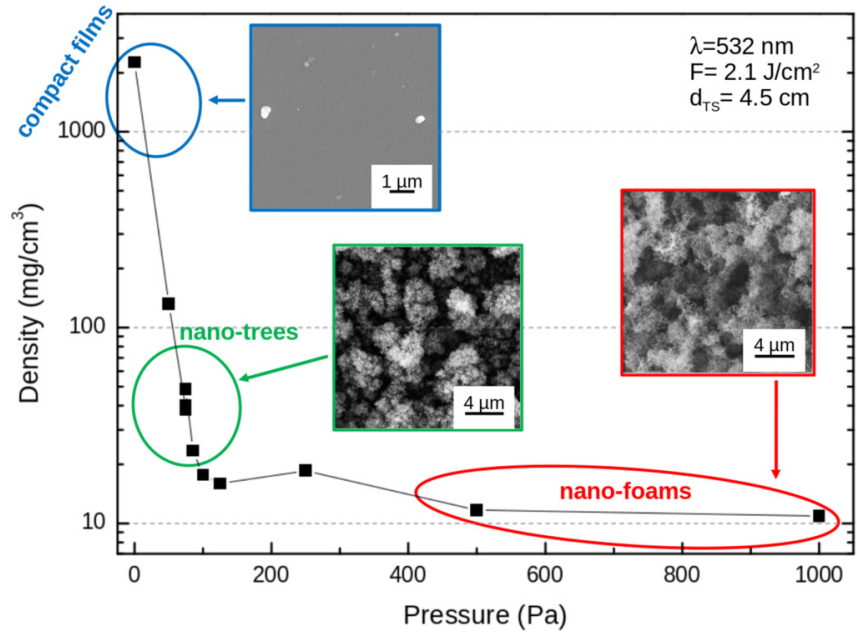

FIG. 1. Average density of PLD carbon films as a function of the background argon pressure. Target to substrate distance is $4.5 \mathrm{~cm}$, laser fluence is $2.1 \mathrm{~J} / \mathrm{cm}^{2}$, repetition rate is $10 \mathrm{~Hz}$.

morphological characteristics of the deposited film. As a general rule valid for typical PLD experiments, the higher the background pressure the lower the density of the resulting film.

In Fig. 1 the average density (measured by the EDXS method, see below) of PLD carbon films is plotted as a function of the background argon pressure for a specific value of laser fluence; the insets show the typical morphology of PLD carbon films for different pressure regimes. Depositions in vacuum or at very low pressure $(<10 \mathrm{~Pa})$ give rise to compact amorphous carbon films, with density close to that of bulk graphite [25]. As the pressure increases the density sharply falls and cauliflowerlike and treelike structures appear [48,49]. Hierarchical nanostructures with similar morphologies have been observed also for different PLD conditions and materials [50]. For pressures exceeding $\sim 100 \mathrm{~Pa}$, the foamlike growth regime, characterized by a density in the range of $10-20 \mathrm{mg} / \mathrm{cm}^{3}$ and a mild dependency of density on pressure, sets in. This deposition regime is characterized by a plume length $\ell_{p}$-defined as the maximum length of the light emitting region during the expansion of the ablated species-shorter than the target-to-substrate distance (for a fluence of $1.6 \mathrm{~J} / \mathrm{cm}^{2}$ and pressure of $700 \mathrm{~Pa} \mathrm{Ar}, \ell_{p} \sim 2 \mathrm{~cm}$ ). This so-called out-of-plume regime implies that the ablated material gets purely diffusional motion before reaching the substrate [51].

The PLD experiments herein reported are performed using the second harmonic $\lambda=532 \mathrm{~nm}$ of a Q-switched Nd:YAG laser (pulsed duration $=5-7 \mathrm{~ns}$ ). The laser can operate in single-shot mode or with a repetition rate equal to $1,2,5$, or $10 \mathrm{~Hz}$. The laser beam is focused on a pyrolitic graphite target with an incidence angle of $45^{\circ}$. Unless otherwise specified, laser fluence is fixed to $1.6 \mathrm{~J} / \mathrm{cm}^{2}$ and the background pressure is $700 \mathrm{~Pa}$ of Ar. During the laser ablation in a high background pressure the target may get covered by ablated material that redeposit in the region nearby the laser spot. These redeposits could be mobilized by subsequent shots and reach the substrate. In order to avoid this phenomenon, the target is 
kept fixed during each experimental run, and is laser cleaned between one experiment and the other. All the depositions are performed at room temperature.

Sample morphology is characterized with a Zeiss Supra 40 field emission scanning electron microscope (SEM, accelerating voltage $3-7 \mathrm{kV}$ ). The electron microscope setup is also used to check deposit composition via energy-dispersive $\mathrm{x}$-ray spectroscopy (EDXS, accelerating voltage 5-10 kV). In addition, EDXS is exploited to calculate the nanofoam areal density: a theoretical model, describing electron transport in the nanofoam/substrate system and characteristic $\mathrm{x}$-ray emission, is used to retrieve the areal density from the measurement of the $K_{\alpha}$ peak intensity of carbon (relative to the nanofoam) and silicon (relative to the substrate) $[52,53]$. The average density of the nanofoam is then calculated as the ratio of the areal density, obtained with EDXS, and the average thickness, measured by SEM cross section images.

Raw grayscale SEM images are processed with a MATLAB ( ${ }^{\circ}$ code developed ad hoc to extract quantitative information about the aggregate morphology and size. The fractal dimension of a single aggregate is extracted with a power spectrum method, which consists of the calculation of the power spectral density $S$, obtained as the squared Fourier transform of the image. This function depends on the radial spatial frequency $q$ with a power-law $S(q) \propto q^{-f^{\prime}}$, where $f^{\prime}$ represent an estimation of the fractal dimension even in the case of noisy and blurred images [54]. In addition, the code converts the grayscale SEM image into a black and white map and then counts each connected white pixel region as an "aggregate" with a certain area $A_{i}$. An equivalent radius $R_{i}$ is assigned to each aggregate by inverting the relation $A_{i}=\pi R_{i}^{2}$. Three quantities are extracted from each processed image: the total number of aggregates $\left(N_{\text {agg }}\right)$, the total coverage $\left(\sum_{i}^{N_{\text {agg }}} A_{i}\right)$, and the averaged equivalent radius $\left(\frac{1}{N_{\text {agg }}} \sum_{i}^{N_{\text {agg }}} R_{i}\right)$. Several SEM images are processed for each sample, and mean values are taken by averaging different images having the same magnification (magnification factor 5000 or 20000 ). Unless otherwise specified, error bars correspond to the standard deviation over each image set. In the following, we consider the power-law exponent $f^{\prime}$ and the average equivalent radius $\overline{R_{\text {eq }}}$ as reasonable estimations of the "real" aggregate fractal dimension $f$ and gyration radius $\overline{R_{g}}$, respectively. As a matter of fact, $f^{\prime}$ and $\overline{R_{\mathrm{eq}}}$ are calculated from two-dimensional images, while real aggregates are three-dimensional objects. Another issue is related to the finite spatial resolution of SEM images, since the pixel size can be as large as $75 \mathrm{~nm}$ (for 5k magnification). Nevertheless, these limitations do not significantly affect the interpretation of the experimental results, at least as far as the the scopes of this paper are concerned $[55,56]$.

\section{RESULTS AND DISCUSSION}

\section{A. Morphology evolution with increasing shots and different substrates}

In the first place we follow the evolution of the morphology of the nanofoam as the number of laser shots increases. An overview of the evolution of aggregate morphology as

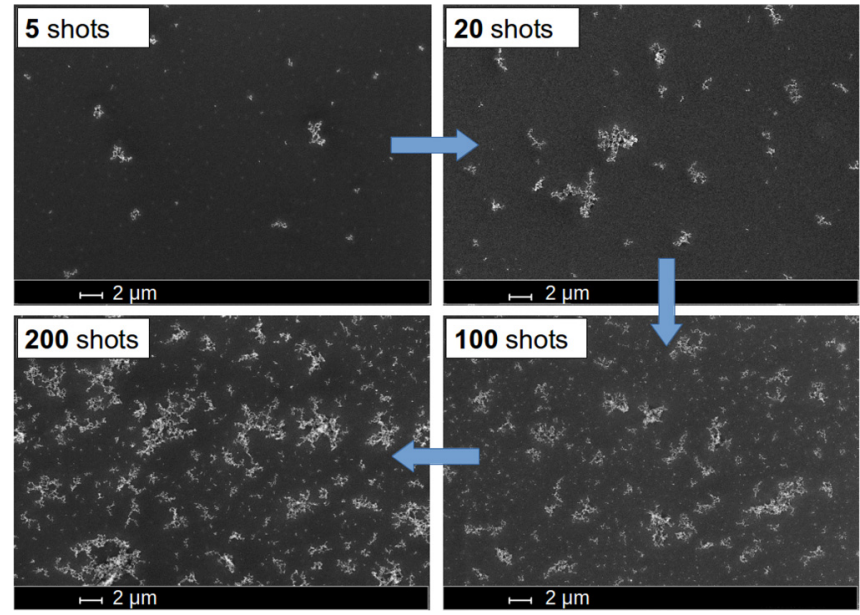

FIG. 2. SEM images of the deposited $\mathrm{C}$ aggregates as a function of the number of laser shots. Background pressure is $700 \mathrm{~Pa} \mathrm{Ar}$, target to substrate distance is $4.5 \mathrm{~cm}$, laser fluence is $1.6 \mathrm{~J} / \mathrm{cm}^{2}$, repetition rate is $10 \mathrm{~Hz}$.

a function of the number of laser shots is given in Fig. 2. Micrometric-sized aggregates of nanoparticles are already present on the substrate even for very few (5-10) shots. An example of the typical aggregate morphology is shown in Fig. 3(a). The average nanoparticle size is around 10-20 nm. The average fractal dimension $f=1.81$ (standard deviation $\sigma_{f}=0.21$ ) is calculated via the Fourier spectrum method [Fig. 3(b)] on a set of 16 SEM images of different aggregates. This value is typical of diffusion-limited cluster-cluster aggregation, in contrast with reaction-limited aggregation processes that usually yield a substantially higher fractal dimension $(f \geqslant 2.1)$ [57,58]. The inherent three-dimensional nature of the aggregates can be appreciated in Fig. 3(c). Since the height of some aggregates is comparable with their lateral size and much larger than the nanoparticle size, a two-dimensional aggregation process-such as diffusion-limited aggregation on substrate-is unlikely.

Additional arguments against on-substrate aggregation can be found considering the evolution of aggregate morphology with the number of laser shots. In a scenario dominated by the aggregation on substrate, one should observe that the average aggregate radius increases with the number of shots and the total coverage [59]. As shown in Fig. 4, the aggregate size seems to be independent from the number of shots and from the total coverage (since the coverage is $\propto R_{g}{ }^{2} \times N_{\text {agg }}$ ). On the other hand, $N_{\text {agg }}$ increases almost linearly with the number of shots, until the aggregates start to coalesce together into a weblike or foamlike structure. These features indicate that most of the ablated material reaches the substrate already in the form of micrometric aggregates, and therefore one must conclude that the aggregation process occurs in flight, i.e., in the gas-filled vacuum chamber before the landing of the aggregates onto the substrate.

To substantiate this claim, we performed additional depositions of substrates other that silicon, namely a $300 \mathrm{~nm}$ thick amorphous carbon $(\mathrm{aC})$ film and a $1 \mu \mathrm{m}$ thick rhodium $(\mathrm{Rh})$ film. We selected these substrates owing to their potential applicative interest (see for example [49]) and the 

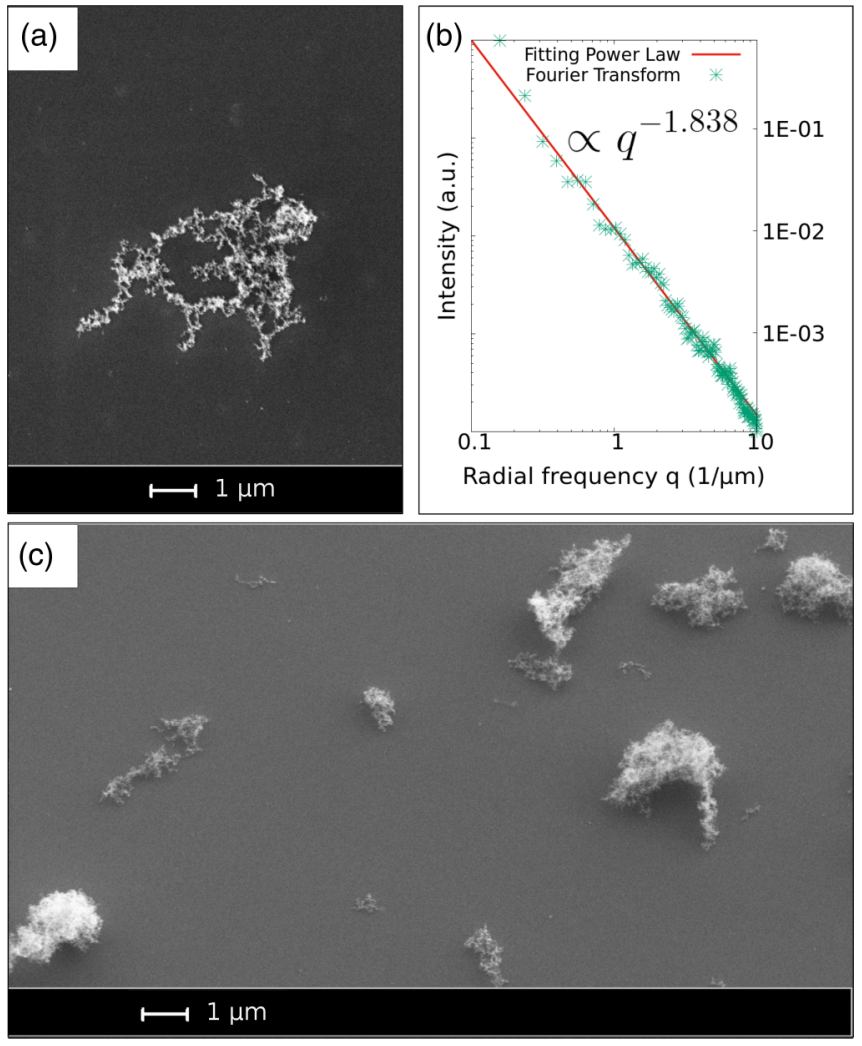

FIG. 3. (a) SEM image of a typical aggregate taken from a 10 shot sample. (b) The aggregate fractal dimension is calculated by fitting the Fourier transform spectrum with a power law of the radial spatial frequency. For the aggregate shown to the right, $f \approx$ 1.84. (c) SEM image of $\mathrm{C}$ aggregates taken with type II secondary electrons (SE2) and imposing a $25^{\circ}$ tilt to the sample holder. Threedimensional nature of the aggregates can be appreciated.

fact that they present important differences in their physical, chemical, and morphological characteristics compared with single crystal silicon. The aC film are deposited in vacuum, in the same experimental configuration (i.e., same target, laser wavelength, laser fluence) employed for the nanofoam depositions, without breaking the vacuum between the compact carbon film growth and the nanofoam depositions. The $\mathrm{Rh}$ film depositions are carried out in vacuum, with $\lambda=1064 \mathrm{~nm}$ and fluence $1.8 \mathrm{~J} / \mathrm{cm}$. The $\mathrm{Rh}$ substrates are not stored in vacuum between their fabrication and the nanofoam deposition. Details about $\mathrm{Rh}$ film production are given in [60]. Both films have been grown with the same PLD setup onto the same silicon slides used as substrate in the other depositions hereby reported. Figure 5 shows how the morphology of aggregates deposited onto silicon [Fig. 5(a)], amorphous carbon [Fig. 5(b)], and rhodium [Fig. 5(c)] is very similar despite the differences among the substrate properties, supporting the hypothesis of in-flight aggregation. We also note that the aggregation process does not seem to be affected by the chemical affinity between substrate and nanoparticles (as in the case of aC substrate) or by the presence of droplets on the Rh surface that could have acted as nucleation seeds if diffusion-limited aggregation on substrate were the dominant aggregation mechanism at play.

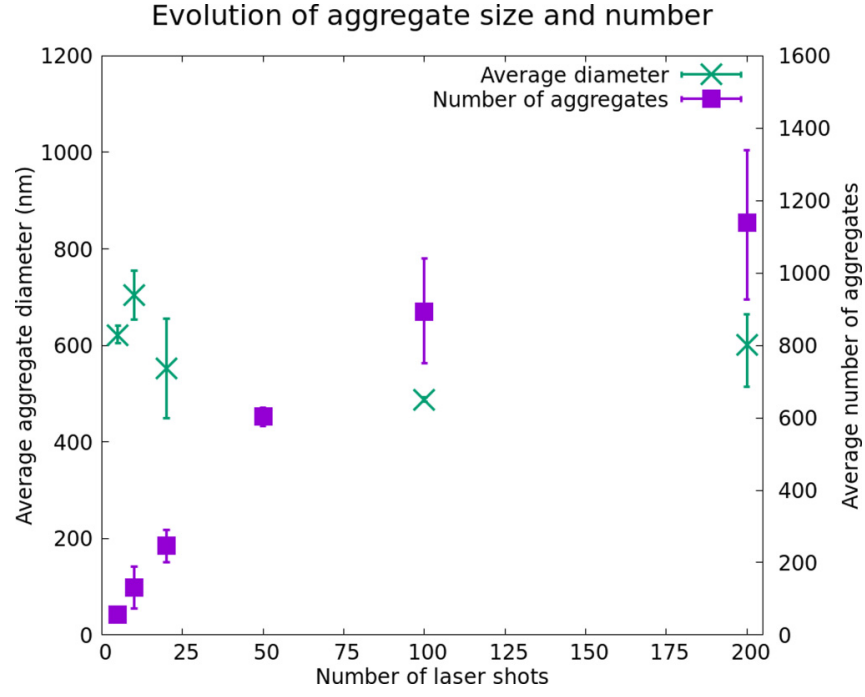

FIG. 4. Number of aggregates (violet squares) and average aggregate radius (green crosses) as a function of the number of laser shots. Background pressure is $700 \mathrm{~Pa} \mathrm{Ar}$, target to substrate distance is $4.5 \mathrm{~cm}$, laser fluence is $1.6 \mathrm{~J} / \mathrm{cm}^{2}$, repetition rate is $1 \mathrm{~Hz}$.

We note that this result differs from the surface diffusionlimited aggregation model proposed in $[46,47]$ to explain the aggregation of gold and titania nanoparticles in femtosecond PLD experiments. On the other hand, it substantiates the claim put forward in [44], where the fractal structure obtained by subpicosecond PLD of iron is attributed to an in-flight clustercluster aggregation process.

This description of the nanofoam growth bears some similarities to what happens during a snowfall: a low density nanofoam layer (the snow blanket) grows because of the coalescence of fractal, void-rich aggregates (the snowflakes). Each aggregate increases its size by clustering of smaller nanoparticles (supercooled water droplets) while is free to float in a nanoparticle-rich atmosphere (supersaturated air masses). The characteristic timescale of the aggregation dynamics $\tau_{\text {agg }}$ corresponds to the amount of time that an aggregate (snowflake) spends in the PLD background atmosphere (earth atmosphere) before being finally deposited on the substrate (the ground).

Given that a snowfall-like process is responsible for the nanofoam growth, one can ask what is the aggregation timescale $\tau_{\text {agg }}$ in a PLD experiment, how $\tau_{\text {agg }}$ determines the properties of the aggregates, and how it can be controlled by acting on the PLD process parameters.

It is well known from the theory of fractal aggregation $[57,61]$ that the aggregation kinetics possesses some universal features that can be expressed in the form of scaling laws between the aggregates properties and the aggregation time. The average size of the aggregates must be a monotonically increasing function of the aggregation time $\tau_{\text {agg }}$; in particular the average gyration radius $\overline{R_{g}}$ should depend on the aggregation time following a power law:

$$
\overline{R_{g}} \propto \tau_{\text {agg }}^{b},
$$

where $b$ is some power-law exponent whose value depends on the specific aggregation mechanism at play $[57,61,62]$. 

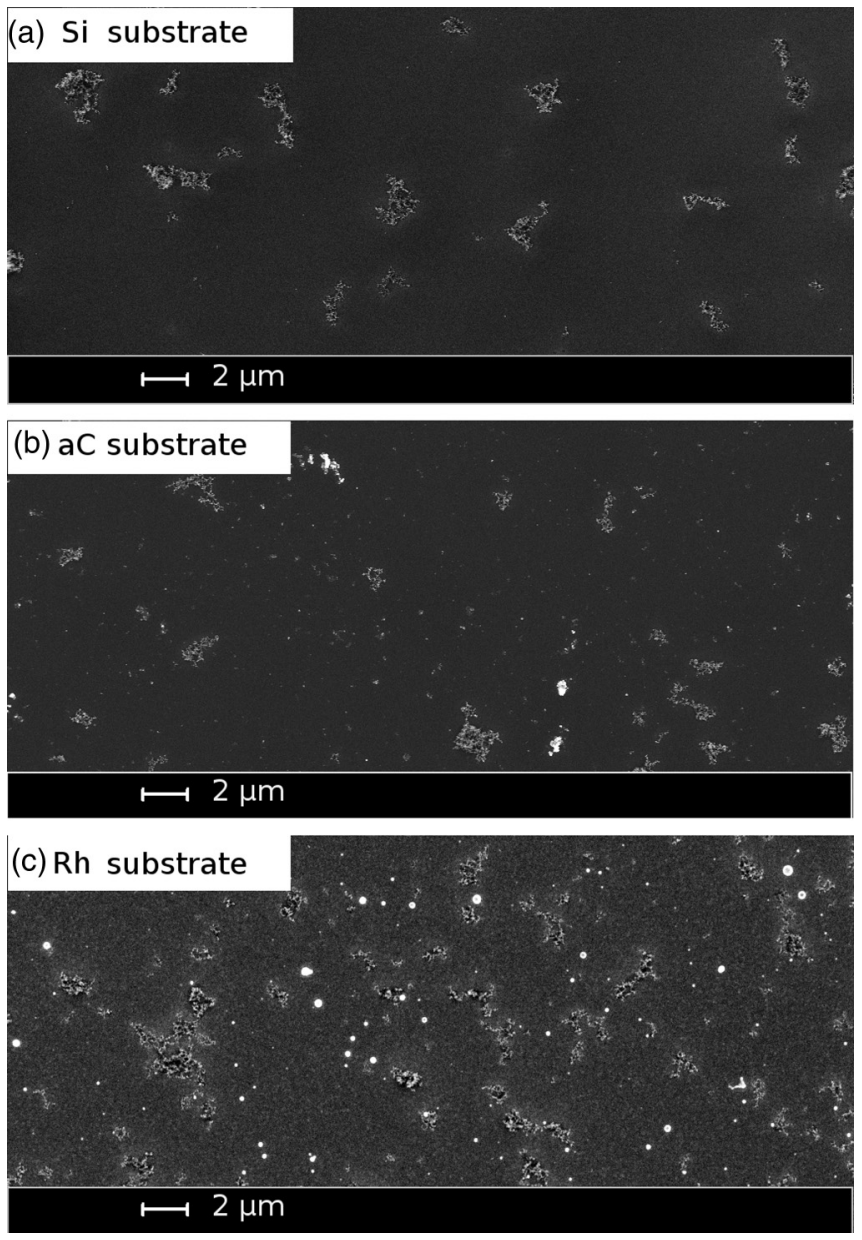

FIG. 5. SEM images of the deposited $\mathrm{C}$ aggregates after 10 laser shots for: (a) silicon substrate, (b) amorphous carbon substrate, and (c) rhodium substrate. The bright, spherical features observed in the case of Rh substrate are droplets of molten Rh ejected by the target during the $\mathrm{Rh}$ film deposition; the textured background is due to the different $\mathrm{Rh}$ crystallite orientation. Background pressure is $700 \mathrm{~Pa}$ Ar, target to substrate distance is $4.5 \mathrm{~cm}$, laser fluence is $1.7 \mathrm{~J} / \mathrm{cm}^{2}$, repetition rate is $1 \mathrm{~Hz}$.

Following the discussion proposed in [61], the exponent can be expressed as $b=z / f$, where $f$ is the fractal dimension and $z$ is a coefficient that accounts for the aggregate mobility. $z=$ $3=z_{\max }$ corresponds to a diffusion coefficient independent from aggregate properties, whereas $z=0.33=z_{\min }$ describes a situation in which the diffusion coefficient goes as the cubic root of the aggregate mass. $z=1$ is a typical value for a diffusion coefficient that scales as the aggregate mass to the $-1 / f$ power, i.e., as $D \propto m^{-1 / f}$.

In summary, one should expect a power-law dependence of the aggregate size on the characteristic aggregation time, with an exponent $b$ which can be related to the fractal dimension as follows:

$$
\frac{z_{\min }}{f}<b<\frac{z_{\max }}{f} \Rightarrow 0.18<b<1.67 .
$$

In the following sections we propose and test two different hypotheses about the aggregation timescale $\tau_{\text {agg }}$, each one related to a different physical description of the aggregation process. First, we suppose that the aggregation timescale is close to the aggregate time-of-fight, showing that under reasonable assumptions $\tau_{\mathrm{agg}}$ (and hence $\overline{R_{g}}$ ) should depend on the target-to-substrate distance as in a power law. The second hypothesis is that the aggregation timescale is given by the shot-to-shot time $T_{\mathrm{sts}}$, which implies that $\overline{R_{g}} \propto\left(T_{\mathrm{sts}}\right)^{b}$. As will be detailed in the next sections, experiments clearly favor the second hypothesis over the first one.

\section{B. Role of the target-to-substrate distance}

A possible ansatz is to assume that $\tau_{\text {agg }}$ is of the same order of the time-of-flight, i.e., the time that an aggregate spends, on average, traveling from the region of nanoparticles nucleation to the substrate. Under this assumption, $\tau_{\mathrm{agg}}$ and hence $\overline{R_{g}}$ must depend in some way on the target-to-substrate distance $d_{\mathrm{ts}}$. It is not trivial to determine the exact scaling relation existing between $\tau_{\text {agg }}$ and $d_{\mathrm{ts}}$. Nonetheless, one can think of two limiting scenarios describing the motion of the aggregates: pure advection (defined by a timescale $\tau_{\text {adv }}$ ) and pure diffusion (timescale $\tau_{\text {diff }}$ ). If both advection and diffusion are present (as one can expect to happen in PLD experiments), the actual aggregation timescale ranges between these limiting values: $\tau_{\mathrm{agg}} \in\left[\tau_{\mathrm{adv}}, \tau_{\mathrm{diff}}\right]$.

In the pure advection limit each aggregate experiences a Stoke's drag force, so that the aggregate speed $v$ asymptotically reaches the fluid velocity of the background gas $u_{f}$ within a timescale $\tau_{\text {drag }}$ :

$$
v(t)=u_{f}\left(1-e^{-\frac{t}{\tau_{\mathrm{drag}}}}\right),
$$

where $\tau_{\text {drag }}=\frac{m}{6 \pi \eta R_{g}}$ depends on the aggregate mass $m$, the gyration radius $R_{g}$, and the dynamic viscosity of the background gas viscosity $\eta$. Once this dynamics has taken place (i.e., for $t \gg \tau_{\text {drag }}$ ), one can assume that the aggregates are at rest in the fluid reference system and thus reach the substrate within the fluid motion timescale $\tau_{f}$, i.e., the time required to cover a distance in the order of $d_{\mathrm{ts}}$ with the same speed as the fluid velocity $u_{f}$. The advection limit is therefore defined by the following relation:

$$
\tau_{\mathrm{adv}}=\tau_{\mathrm{drag}}+\tau_{f} .
$$

The timescale of the drag force $\tau_{\text {drag }}$ can be estimated by making use of the fractal scaling [63], i.e., by rewriting the aggregate mass in terms of nanoparticle radius $R_{\mathrm{np}}$, nanoparticle density $\rho_{\mathrm{np}}$, and fractal dimension $f$ :

$$
m=\frac{4}{3} \pi \rho_{\mathrm{np}} R_{g}^{f} R_{\mathrm{np}}^{3-f} .
$$

Taking $\eta=2.3 \times 10^{-5} \quad$ Pa $\quad$ s, $\quad R_{\mathrm{np}}=20 \mathrm{~nm}, \quad \rho_{\mathrm{np}}=$ $2 \mathrm{mg} / \mathrm{cm}^{3}$ as the dynamic viscosity of argon at room temperature, the average nanoparticle radius [25], and the density of pulsed laser deposited amorphous carbon nanoparticles [64], respectively, the drag force timescale varies from $\tau_{\mathrm{drag}} \approx 8 \mathrm{~ns}$ in the case of a single nanoparticle $\left(R_{g}=R_{\mathrm{np}}\right)$ up to $\tau_{\mathrm{drag}} \approx 700 \mathrm{~ns}$ for an aggregate like the one shown in Fig. $3\left(R_{g} \approx 4 \mu \mathrm{m}, f \approx 1.8\right)$. In other words, $\tau_{\text {drag }}$ is always in the order of a microsecond or less. The timescale of the fluid motion $\tau_{f}$ depends on the fluid velocity field $u_{f}$. Assuming that the fluid motion of the background 
gas is determined by the propagation of the laser-generated shockwave, we have that $u_{f}$ corresponds to the velocity of a shocked fluid; in this picture the aggregates are dragged by the wake of the laser shockwave. In principle, the fluid velocity after the passage of a shock can be derived from Rankine-Hugoniot relations; exact analytical solutions, however, can be only found under strong simplifying assumption [65]. For the purpose of finding a scaling law between $\tau_{\text {agg }}$ and $d_{\mathrm{ts}}$ we can neglect the exact fluid velocity profile and simply consider $u_{f}=c_{s} / k$, where $c_{s}=319 \mathrm{~m} / \mathrm{s}$ is the speed of sound in Ar at room temperature and $k>1$ is some factor few times the unity, obtaining

$$
\tau_{f} \approx k \frac{d_{\mathrm{ts}}}{c_{s}} .
$$

Since $d_{\mathrm{ts}}$ is in the order of few centimeters and $k>1, \tau_{f}>$ $200 \mu \mathrm{s} \gg \tau_{\mathrm{drag}}$, and the following relation holds:

$$
\tau_{\mathrm{adv}}=\tau_{f}+\tau_{\mathrm{drag}} \approx \tau_{f} \approx k \frac{d_{\mathrm{ts}}}{c_{s}} .
$$

By putting Eq. (7) in the scaling law of Eq. (1) one found that, in the advection limit $\tau_{\text {agg }} \approx \tau_{\text {adv }}$, the average aggregate radius should depend on the target-to-substrate distance as

$$
\overline{R_{g}} \propto\left(d_{\mathrm{ts}}\right)^{b} .
$$

On the other hand, in the pure diffusion limit, the aggregate motion can be thought of as a Brownian motion. The mean squared displacement from the region of nanoparticle synthesis can be expressed in terms of the time elapsed $t$ and the diffusion coefficient $D$ as $\overline{x^{2}}=2 D t[66,67]$. In other words, the timescale of the diffusive motion toward the substrate, which coincides with the time available for aggregation, goes as the square of the target-to-substrate distance $\tau_{\text {diff }}=\frac{\left(d_{\mathrm{ts}}\right)^{2}}{2 D}$. Therefore, in the pure diffusion limit $\left(\tau_{\mathrm{agg}}=\tau_{\mathrm{diff}}\right)$ one has

$$
\tau_{\text {agg }}=\frac{\left(d_{\mathrm{ts}}\right)^{2}}{2 D}
$$

Which, together with Eq. (1), implies

$$
\overline{R_{g}} \propto\left(d_{\mathrm{ts}}\right)^{2 b} .
$$

By comparing Eqs. (8) and (10) we conclude that the timeof-flight ansatz should imply a power-law relation $\overline{R_{g}} \propto d_{\mathrm{ts}}{ }^{\prime}$ with an exponent $b^{\prime} \in[b, 2 b]$, where the equalities $b^{\prime}=b$ and $b^{\prime}=2 b$ represent the pure advection and the pure diffusion limits, respectively. Using the fractal scaling for $b$ described by Eq. (2) one has

$$
\overline{R_{g}} \propto d_{\mathrm{ts}}^{b^{\prime}}, \quad 0.18<b^{\prime}<3.34 .
$$

This hypothesis has been tested by performing different depositions with $d_{t s}=35,45,55$, and $65 \mathrm{~mm}$, respectively, all the other parameters being fixed. Typical aggregate morphologies are shown in Fig. 6. We first note that the number of aggregates and the areal coverage decrease sharply with increasing $d_{\mathrm{ts}}$. This can be understood considering that with increasing $d_{\mathrm{ts}}$ the solid angle intercepted by the substrate becomes smaller, and thus the quantity of aggregates deposited per surface unit is reduced.

Figure 7 shows the normalized coverage (violet squares) and the average aggregate radius (green crosses) as a function
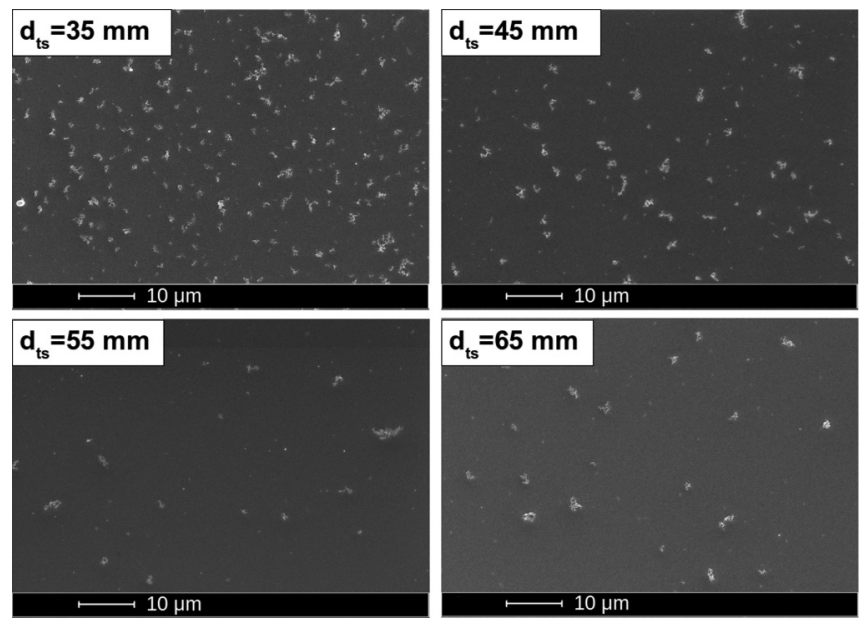

FIG. 6. SEM images of the deposited aggregates as a function of the target to substrate distance. Each deposition is performed with 10 shots, background pressure of $700 \mathrm{~Pa} \mathrm{Ar}$, laser fluence $1.6 \mathrm{~J} / \mathrm{cm}^{2}$, repetition rate $1 \mathrm{~Hz}$.

of $d_{\mathrm{ts}}$. The value of $\overline{R_{g}}$ appears to be independent from $d_{\mathrm{ts}}$ within the error bars, in contrast with Eq. (11) which predicts a power-law dependence on $d_{\mathrm{ts}}$. Moreover, the power-law function that best fits the experimental data (red solid line in Fig. 7) has an exponent $b^{\prime} \approx 0.13$, outside the range of expected values [see Eq. (11)]. Therefore, one must conclude that the main timescale of the aggregation kinetics is not given by the time-of-flight of the aggregates. Also, we note that the very weak dependence (if any) of the aggregate size on their number (and hence on the areal coverage) is not compatible

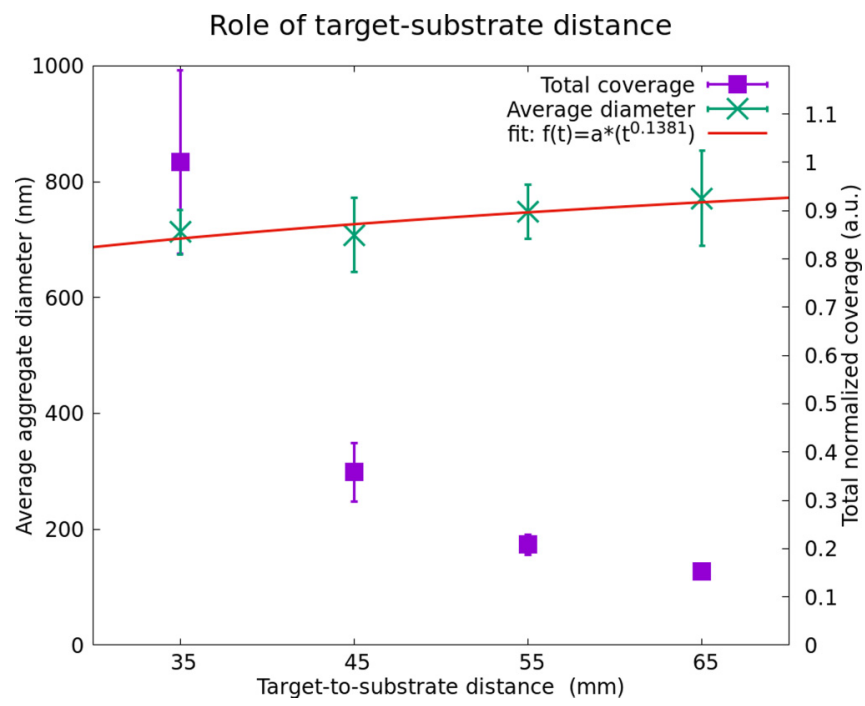

FIG. 7. Violet squares: Average areal coverage (normalized to its highest value) as a function of $d_{\mathrm{ts}}$. Green crosses: Average aggregate radius as a function of $d_{\mathrm{ts}}$. Red solid line: Fitting power-law curve $f=a\left(d_{\mathrm{ts}}\right)^{b^{\prime}}$ for the average radius. $a$ and $b^{\prime}$ are obtained with with a Levenberg-Marquardt least square algorithm. Background pressure is $700 \mathrm{~Pa}$ Ar, laser fluence $1.6 \mathrm{~J} / \mathrm{cm}^{2}, 1 \mathrm{~Hz}$ repetition rate, and 10 laser shots. 
with diffusion-limited aggregation mechanisms occurring on the substrate surface, as those observed in $[46,47]$.

\section{Role of the shot-to-shot time}

The results presented in the previous section suggest that there must be another characteristic timescale which is relevant in determining the value of $\tau_{\text {agg }}$. One possibility is that the characteristic timescale of the aggregation dynamics is determined by two additive contributions, namely the timeof-flight of the aggregates plus the time interval between two consecutive laser shots $\left(T_{\text {sts }}\right)$. This hypothesis corresponds to a physical scenario where the nanoparticles generated by the $n$th laser shots are free to aggregate in the vacuum chamber for a timescale $T_{\text {sts }}$ until the shockwave generated by the subsequent $(n+1)$ th laser shot drags them toward the substrate within a timescale $\tau_{\text {adv }}$, i.e., $\tau_{\text {agg }}=T_{\text {sts }}+\tau_{\text {adv }}$.

In the previous section we showed that $\tau_{\text {adv }}=\tau_{\text {drag }}+\tau_{f}$ is in the order of few milliseconds or less, while in our experiments $T_{\text {sts }}$ (i.e., the inverse of laser repetition rate) ranges from 0.1 to $10 \mathrm{~s}$. Therefore, given that $\tau_{\text {drag }} \ll \tau_{f} \ll T_{\text {sts }}$, Eq. (7) can be modified as follows:

$$
\tau_{\text {agg }}=\tau_{\text {drag }}+\tau_{f}+T_{\text {sts }} \approx T_{\text {sts }} .
$$

From Eqs. (1) and (2) it follows immediately that

$$
\overline{R_{g}} \propto\left(T_{\text {sts }}\right)^{b}, \quad 0.18<b<1.67 .
$$

In the light of the snowfall analogy, this hypothesis is equivalent to say that the size of a snowflake is determined by the amount of time it spends floating in the supercooled atmosphere rather then the amount of time it takes to fall to the ground, being the latter is much smaller than the former. Incidentally, this mechanism is quite similar to the one at play in actual snowfalls [68].

We tested this prediction in PLD experiments by varying the laser repetition rate from 10 to $0.2 \mathrm{~Hz}$, to which corresponds $T_{\mathrm{sts}}=0.1,0.2,0.5,1,2$, and $5 \mathrm{~s}$, while keeping fixed all the other process parameters. An example of the aggregate morphology is given is Fig. 8 .

The corresponding value of the average aggregate radius (green crosses) and surface coverage (violet squares) are given in Fig. 9.

The red solid line in Fig. 9 is the power law in the form of Eq. (13) that best fits the experimental data for the value of $\overline{R_{g}}$ as a function $T_{\text {sts. }}$. The fitting is in excellent agreement with the data, and the exponent $b \approx 0.27$ lies within the range derived by Eqs. (2) and (13); the hypothesis $\tau_{\mathrm{agg}} \approx T_{\mathrm{sts}}$ is thereby validated.

We point out that the presence of a shot-to-shot timescale is a specific and intrinsic feature of the PLD technique, not shared with other deposition techniques commonly employed for the synthesis of nanostructured materials such as magnetron sputtering or supersonic cluster beam deposition. In fact, as long as the inequality $T_{\text {sts }} \gg \tau_{f} \approx 1$ ms holds true, the observed relationship between the aggregate size and the laser repetition rate can be exploited as a major (and largely unexplored so far) degree of freedom in the choice of the optimal PLD parameters for tailoring the morphological properties of ultralow density foams. Indeed, the foams grown from the coalescence of larger aggregates should be characterized by a
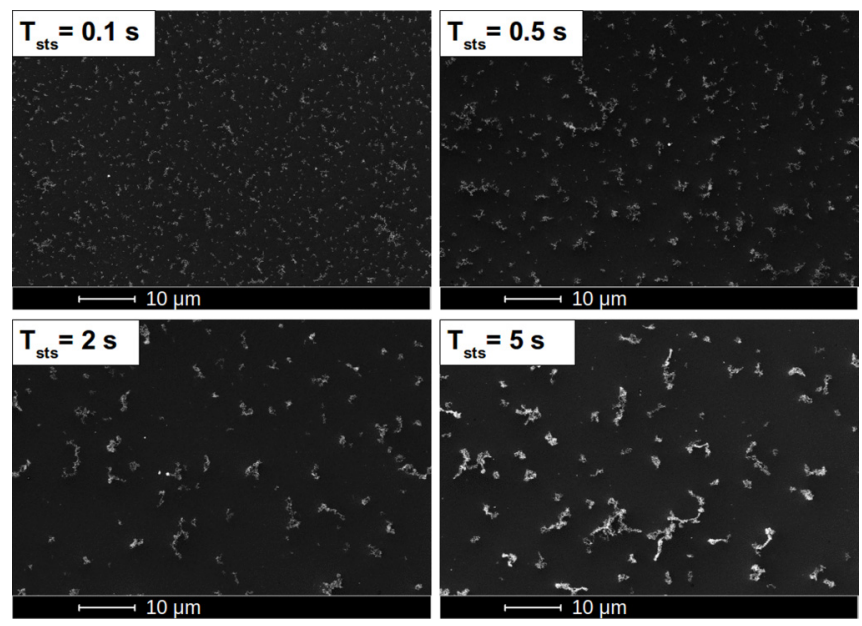

FIG. 8. SEM images of the deposited aggregates as a function of the shot-to-shot time $T_{\text {sts }}$. Each deposition is performed with 10 shots, background pressure of $700 \mathrm{~Pa} \mathrm{Ar}$, laser fluence $1.7 \mathrm{~J} / \mathrm{cm}^{2}$, target to substrate distance $4.5 \mathrm{~cm}$.

looser packing of the aggregate themselves. This is turn may result in a reduction of short-range uniformity and a lower average density. We demonstrate this approach by performing two PLD depositions (7000 shots each) with different laser repetition rate (namely 1 and $10 \mathrm{~Hz}$ ), all the other parameters being fixed. The morphology of the resulting nanofoams is shown in Fig. 10, along with a density map performed with the EDXS method [52,53]. The nanofoam grown with $T_{\text {sts }}=0.1 \mathrm{~s}$ (bottom) presents a more uniform morphology compared to the one grown with $T_{\text {sts }}=1 \mathrm{~s}$ (top). The average density is $\rho \approx 18.7 \mathrm{mg} / \mathrm{cm}^{3}$ for $T_{\text {sts }}=0.1 \mathrm{~s}$ and $\rho \approx 14.3 \mathrm{mg} / \mathrm{cm}^{3}$ for

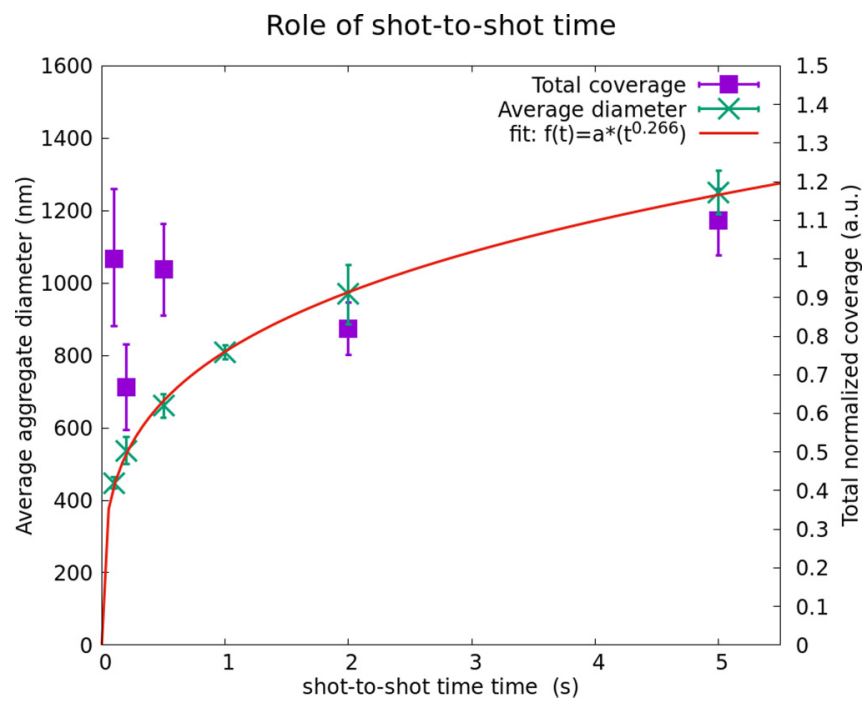

FIG. 9. Violet squares: Average areal coverage (normalized to the first value) as a function of $T_{\text {sts }}$. Green crosses: Average aggregate radius as a function of $T_{\text {sts }}$. Red solid line: Fitting power-law curve $f=a\left(T_{\mathrm{sts}}\right)^{b^{\prime}}$ for the average radius. $a \approx 810$ and $b^{\prime} \approx 0.27$ are obtained with with a Levenberg-Marquardt least square algorithm. Background pressure is $700 \mathrm{~Pa} \mathrm{Ar}$, laser fluence $1.7 \mathrm{~J} / \mathrm{cm}^{2}, d_{t s}=$ $4.5 \mathrm{~cm}$, and 10 laser shots. 

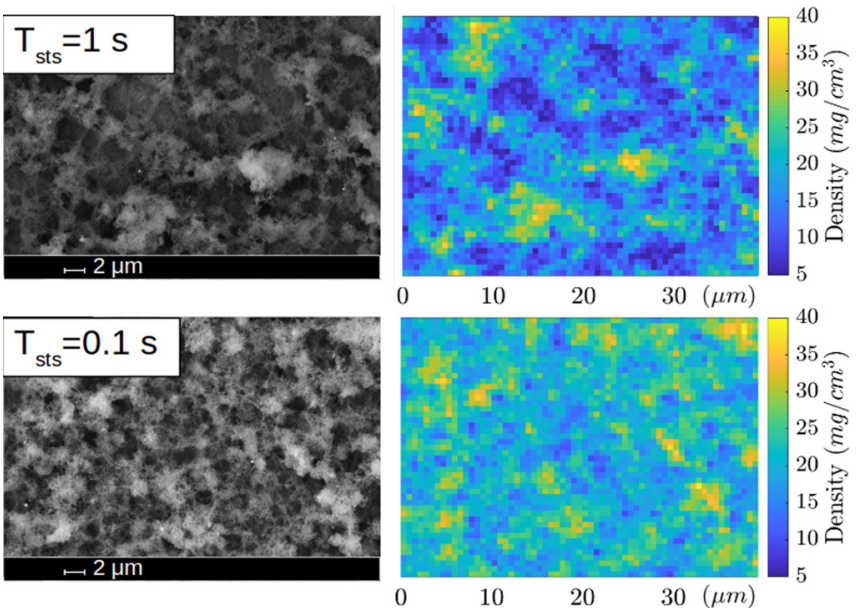

FIG. 10. SEM images (left side) and EDXS density maps (right side) of two carbon foams deposited with $T_{\text {sts }}=1 \mathrm{~s}$ (top) and $T_{\text {sts }}=$ $0.1 \mathrm{~s}$ (bottom). Each deposition is performed with 7000 shots, background pressure of $1000 \mathrm{~Pa} \mathrm{Ar}$, laser fluence $2.1 \mathrm{~J} / \mathrm{cm}^{2}$, target to substrate distance $4.5 \mathrm{~cm}$.

$T_{\mathrm{sts}}=1 \mathrm{~s}$, thus confirming that a proper selection of the laser repetition rate can allow a fine tuning of the morphological features of low-density, nanostructured materials.

\section{CONCLUSION}

In conclusion, in this paper we propose a snowfall-like model to describe the growth dynamics of nanostructured, ultralow density material in pulsed laser deposition experiments. According to this model, the nanofoam peculiar morphology is the result of the superposition and coalescence of micrometer-sized aggregates of carbon nanoparticles. The aggregation process occurs in flight, i.e., before the aggregate landing. A power-law scaling exists between aggregate size and the aggregation timescale, and we show that the latter is dominated by the shot-to-shot time. We also demonstrate how this result can be exploited to tailor the nanofoam properties (e.g., density and uniformity) by acting on the laser repetition rate.

This work is focused on the growth of carbon nanofoams, an important class of nanomaterials that finds application in many scientific and technological sectors. Nonetheless, the model we propose is based on very general assumptions, and the experimental method that we employed is suitable for a straightforward generalization to ultralow density materials with different composition. Indeed, a research activity is ongoing to study the growth of other nanomaterials (e.g., copper and titanium oxide) also in different PLD regimes. In particular, the study of nanofoam growth by PLD with femtosecond pulses and $\mathrm{kHz}$ repetition rate represents a natural prosecution of this work, since the mechanism of nanoparticle generation and the shot-to-shot time can differ significantly from the nanosecond, $10 \mathrm{~Hz}$ regime.

\section{ACKNOWLEDGMENTS}

The authors wish to thank Luca Fedeli for useful discussion. The research leading to these results has received funding from the European Research Council Consolidator Grant ENSURE (ERC-2014-CoG No. 647554).
[1] For such highly porous materials, density is considered as a mean value averaged over a length scale sufficiently larger than the typical pore size.

[2] D. A. Gilbert, E. C. Burks, S. V. Ushakov, P. Abellan, I. Arslan, T. E. Felter, A. Navrotsky, and K. Liu, Chem. Mater. 29, 9814 (2017).

[3] G. Guoxin, W. H. Bin, D. Shujiang, L. Li-Min, and L. X. Wen, Small 11, 804 (2014).

[4] N. K. Chaudhari, H. Jin, B. Kim, and K. Lee, Nanoscale 9, 12231 (2017).

[5] S. Fu, J. Song, C. Zhu, G.-L. Xu, K. Amine, C. Sun, X. Li, M. H. Engelhard, D. Du, and Y. Lin, Nano Energy 44, 319 (2018).

[6] L. S. Zhong, J. S. Hu, H. P. Liang, A. M. Cao, W. G. Song, and L. J. Wan, Adv. Mater. 18, 2426 (2006).

[7] M. P. Siegal, D. L. Overmyer, R. J. Kottenstette, D. R. Tallant, and W. G. Yelton, Appl. Phys. Lett. 80, 3940 (2002).

[8] L. Xiaoming, W. Lu, F. Yubo, F. Qingling, C. Fu-Zhai, and W. Fumio, J. Biomed. Mater. Res. Part A 101A, 2424 (2013).

[9] H. Jia, J. An, X. Guo, C. Su, L. Zhang, H. Zhou, and C. Xie, J. Mol. Liq. 212, 763 (2015).

[10] A. Kurek, R. Xalter, M. Stürzel, and R. Mülhaupt, Macromolecules 46, 9197 (2013).

[11] J. A. Grant-Jacob, B. Mills, and R. W. Eason, J. Phys. D 47, 055105 (2014).
[12] T. Iino and K. Nakamura, Jpn. J. Appl. Phys. 48, 07GE01 (2009).

[13] A. V. Rode, E. G. Gamaly, A. G. Christy, J. G. Fitz Gerald, S. T. Hyde, R. G. Elliman, B. Luther-Davies, A. I. Veinger, J. Androulakis, and J. Giapintzakis, Phys. Rev. B 70, 054407 (2004).

[14] D. C. Mattis, Phys. Rev. B 71, 144424 (2005).

[15] V. G. Shvedov, A. V. Rode, Y. V. Izdebskaya, A. S Desyatnikov, W. Krolikowski, and Y. S. Kivshar, Phys. Rev. Lett. 105, 118103 (2010).

[16] R. Blinc, P. Cevc, D. Arčon, B. Zalar, A. Zorko, T. Apih, F. Milia, N. R. Madsen, A. G. Christy, and A. V. Rode, Phys. Status Solidi B 243, 3069 (2006)

[17] M. Roy, in Functional Materials, edited by S. Banerjee and A. Tyagi (Elsevier, London, 2012), pp. 675-706.

[18] A. Rode, S. Hyde, E. Gamaly, R. Elliman, D. McKenzie, and S. Bulcock, Appl. Phys. A 69, S755 (1999).

[19] A. Rode, E. Gamaly, and B. Luther-Davies, Appl. Phys. A 70, 135 (2000).

[20] E. Muñoz, M. de Val, M. L. Ruiz-González, C. López-Gascón, M. L. Sanjuán, M. T. Martínez, J. M. González-Calbet, G. F. de la Fuente, and M. Laguna, Chem. Phys. Lett. 420, 86 (2006).

[21] E. G. Gamaly, N. R. Madsen, D. Golberg, and A. V. Rode, Phys. Rev. B 80, 184113 (2009). 
[22] E. Muñoz, M. L. Ruiz-González, A. Seral-Ascaso, M. L. Sanjuán, J. M. González-Calbet, M. Laguna, and G. F. de la Fuente, Carbon 48, 1807 (2010).

[23] M. Pervolaraki, P. Komninou, J. Kioseoglou, A. Othonos, and J. Giapintzakis, Appl. Surf. Sci. 278, 101 (2013).

[24] A. Macchi, M. Borghesi, and M. Passoni, Rev. Mod. Phys. 85, 751 (2013).

[25] A. Zani, D. Dellasega, V. Russo, and M. Passoni, Carbon 56, 358 (2013).

[26] M. Passoni, A. Zani, A. Sgattoni, D. Dellasega, A. Macchi, I. Prencipe, V. Floquet, P. Martin, T. V. Liseykina, and T. Ceccotti, Plasma Phys. Controlled Fusion 56, 045001 (2014).

[27] M. Passoni, A. Sgattoni, I. Prencipe, L. Fedeli, D. Dellasega, L. Cialfi, I. W. Choi, I. J. Kim, K. A. Janulewicz, H. W. Lee, J. H. Sung, S. K. Lee, and C. H. Nam, Phys. Rev. Accel. Beams 19, 061301 (2016).

[28] I. Prencipe, A. Sgattoni, D. Dellasega, L. Fedeli, L. Cialfi, I. W. Choi, I. J. Kim, K. A. Janulewicz, K. F. Kakolee, H. W. Lee, J. H. Sung, S. K. Lee, C. H. Nam, and M. Passoni, Plasma Phys. Controlled Fusion 58, 034019 (2016).

[29] L. Fedeli, A. Formenti, L. Cialfi, A. Pazzaglia, and M. Passoni, Sci. Rep. 8, 3834 (2018).

[30] Also called by some authors atomic clusters or simply clusters.

[31] S. J. Henley, J. D. Carey, S. R. P. Silva, G. M. Fuge, M. N. R. Ashfold, and D. Anglos, Phys. Rev. B 72, 205413 (2005).

[32] D. B. Geohegan, A. A. Puretzky, G. Duscher, and S. J. Pennycook, Appl. Phys. Lett. 72, 2987 (1998).

[33] S. Palanco, S. Marino, M. Gabás, S. Bijani, L. Ayala, and J. R. Ramos-Barrado, Opt. Express 22, 3991 (2014).

[34] M. Ghidelli, L. Mascaretti, B. R. Bricchi, A. Zapelli, V. Russo, C. S. Casari, and A. L. Bassi, Appl. Surf. Sci. 434, 1064 (2018).

[35] J. Perrière, C. Boulmer-Leborgne, R. Benzerga, and S. Tricot, J. Phys. D 40, 7069 (2007).

[36] S. S. Harilal, N. Farid, A. Hassanein, and V. M. Kozhevin, J. Appl. Phys. 114, 203302 (2013).

[37] G. O'Connell, T. Donnelly, and J. G. Lunney, Appl. Phys. A 117, 289 (2014).

[38] R. Wood, J. Leboeuf, K. Chen, D. Geohegan, and A. Puretzky, Appl. Surf. Sci. 127-129, 151 (1998).

[39] J. Gonzalo, A. Perea, D. Babonneau, C. N. Afonso, N. Beer, J.-P. Barnes, A. K. Petford-Long, D. E. Hole, and P. D. Townsend, Phys. Rev. B 71, 125420 (2005).

[40] M. E. Povarnitsyn, T. E. Itina, M. Sentis, K. V. Khishchenko, and P. R. Levashov, Phys. Rev. B 75, 235414 (2007).

[41] T. E. Itina and L. V. Zhigilei, J. Phys.: Conf. Ser. 59, 44 (2007).

[42] S. Amoruso, G. Ausanio, A. C. Barone, R. Bruzzese, L. Gragnaniello, M. Vitiello, and X. Wang, J. Phys. B 38, L329 (2005).

[43] I. Mirza, G. O’Connell, J. J. Wang, and J. G. Lunney, Nanotechnology 25, 265301 (2014).
[44] K. Nishikawa, K. Takano, H. Miyahara, T. Kawamura, A. Okino, E. Hotta, T. Nayuki, Y. Oishi, T. Fujii, X. Wang, and K. Nemoto, Appl. Phys. Lett. 89, 243112 (2006).

[45] N. E. Kask, E. G. Leksina, S. V. Michurin, and G. M. Fedorov, Laser Phys. 18, 762 (2008).

[46] G. L. Celardo, D. Archetti, G. Ferrini, L. Gavioli, P. Pingue, and E. Cavaliere, Mater. Res. Express 4, 015013 (2017).

[47] E. Cavaliere, G. Benetti, G. L. Celardo, D. Archetti, P. Pingue, G. Ferrini, and L. Gavioli, J. Nanopart. Res. 19, 311 (2017).

[48] A. Uccello, A. Maffini, D. Dellasega, and M. Passoni, Fusion Eng. Des. 88, 1347 (2013), Proceedings of the 27th Symposium On Fusion Technology (SOFT-27), Liège, Belgium, September 24-28, 2012.

[49] A. Maffini, A. Uccello, D. Dellasega, V. Russo, S. Perissinotto, and M. Passoni, J. Nucl. Mater. 463, 944 (2015).

[50] P. Gondoni, V. Russo, C. E. Bottani, A. L. Bassi, and C. S. Casari, MRS Proc. 1497, mrsf121497 (2013).

[51] D. Dellasega, A. Facibeni, F. D. Fonzo, V. Russo, C. Conti, C. Ducati, C. Casari, A. L. Bassi, and C. Bottani, Appl. Surf. Sci. 255, 5248 (2009).

[52] I. Prencipe, D. Dellasega, A. Zani, D. Rizzo, and M. Passoni, Sci. Technol. Adv. Mater. 16, 025007 (2015).

[53] A. Pazzaglia, A. Maffini, D. Dellasega, A. Lamperti, and M. Passoni, Mater. Charact. 153, 92 (2019).

[54] C. M. Sorensen, Aerosol Sci. Technol. 35, 648 (2001).

[55] P. Soille and J.-F. Rivest, J. Visual Commun. Image Represent 7, 217 (1996).

[56] F. Maggi and J. C. Winterwerp, Phys. Rev. E 69, 011405 (2004).

[57] P. Meakin, Phys. Scr. 46, 295 (1992).

[58] A. M. Brasil, T. L. Farias, and M. G. Carvalho, Aerosol Sci. Technol. 33, 440 (2000).

[59] M. Kostoglou and A. G. Konstandopoulos, J. Aerosol Sci. 32, 1399 (2001).

[60] A. Uccello, D. Dellasega, S. Perissinotto, N. Lecis, and M. Passoni, J. Nucl. Mater. 432, 261 (2013).

[61] V. Tamas, Fractal Growth Phenomena (Second Edition) (World Scientific, Singapore, 1992).

[62] M. Y. Lin, H. M. Lindsay, D. A. Weitz, R. Klein, R. C. Ball, and P. Meakin, J. Phys.: Condens. Matter 2, 3093 (1990).

[63] L. de Martín, A. Fabre, and J. R. van Ommen, Chem. Eng. Sci. 112, 79 (2014).

[64] E. Cappelli, C. Scilletta, M. Servidori, V. Valentini, and S. Orlando, Diam. Relat. Mater. 17, 1476 (2008).

[65] As in the case of $1 \mathrm{D}$ geometry or for a strong shock with spherical symmetry, see L. I. Sedov, Similarity and Dimensional Methods in Mechanics (Academic, New York, 1959).

[66] A. Einstein, Ann. Phys. 322, 549 (1905).

[67] F. Reif, Am. J. Phys. 66, 164 (1998).

[68] K. G. Libbrecht, Annu. Rev. Mater. Res. 47, 271 (2017). 